\title{
Editorial: Hybridizing Quantum Physics and Engineering
}

This year marks the 35th anniversary of landmark experiments, reported in Physical Review Letters [1,2], that tested one of the most bewildering predictions of quantum mechanics: Information can exist nonlocally, so that retrieving it requires performing measurements on two distinct particles that are said to be entangled. This prediction had been troubling physicists for decades. The perplexity arose from thought experiments, conceived by Einstein, which illustrated that entanglement would violate the classical premise that the laws of physics should act locally and deterministically $[3,4]$. The advent of laboratory experiments verifying the most revolutionary features of quantum theory marked a turning point in the field. Ever more confident that the theory was correct and complete, quantum scientists reinvented themselves as engineers on a quest to harness entanglement for the advancement of technology.

Today, physicists can manipulate quantum states in myriad media, from optical and microwave photons to laser-cooled ions and atoms, semiconductor quantum dots, and even macroscopic mechanical oscillators. But no single medium can meet all demands for realizing quantum technologies - such as sensors, computers, and secure communication networks - and interfacing them with existing classical infrastructure. Thus, a crucial endeavor is merging the strengths of different media into hybrid quantum systems.

Illustrative of the need for hybrid quantum systems are the complementary strengths of light and matter. Light is an ideal conveyor of quantum information. A quantum bit (qubit) can be encoded in a photon's polarization and rapidly transmitted along a fiber-optic cable. However, photons alone are insufficient to form a switch - they cannot interact with one another directly. Making one photon influence another requires that both interact with an auxiliary medium [5-8], for example, atoms confined near the optical fiber $[7,8]$ down which the photons are traveling. Hybrid systems also enable photons to transfer information into matter-based memories for long-term storage [8-10], or controllably link solid-state qubits for computation [11,12].

Connecting individual quantum processors into larger networks will require additional links in the hybrid chain. Promising building blocks for quantum computers are superconducting qubits that "talk" via their mutual interaction with a microwave field [11,12]. Microwaves, unlike optical photons, cannot be efficiently transmitted over long distances. However, recent experiments show that a microwave signal can be transferred to the optical domain using a mechanical oscillator [13-15]. Transferring quantum information without adding classical noise requires cooling the oscillator into its motional ground state, a difficult but demonstrated feat [16-18].

In the quantum engineer's expanding toolbox, mechanical oscillators stand out for their ability to interact with almost anything. Mechanical oscillators can be tools for preparing nonclassical states of light [19,20] or matter [21], enabling quantum enhancements in interferometry or magnetometry. For example, electronic spins entangled via their mutual coupling to the motion of a diamond cantilever $[21,22]$ could form next-generation magnetometers with an unprecedented combination of sensitivity, spatial resolution, and measurement bandwidth. Conversely, a nonclassical state of the cantilever, preparable with the aid of a single spin, could sensitively probe the interplay between quantum mechanics and gravity $[23,24]$.

Thus, hybrid systems are bringing quantum engineers full circle, back to probing the frontiers of fundamental physics. While quantum theory has passed every test in few-particle systems, open questions lie in the quantum theory of massive objects and of systems with many degrees of freedom. Unanticipated quantum collective behaviors have been discovered in many-electron systems time and again. Will interfacing disparate quantum media [25-27], and incorporating them into complex networks, lead us to discover entirely new emergent phenomena? Hybrid systems are likely to harbor surprises that will fuel quantum science for decades to come.

\author{
Monika Schleier-Smith \\ Department of Physics, \\ Stanford University, \\ Stanford, California 94305, USA
}

Published 29 August 2016

DOI: 10.1103/PhysRevLett.117.100001 
[1] A. Aspect, P. Grangier, and G. Roger, Phys. Rev. Lett. 47, 460 (1981).

[2] A. Aspect, P. Grangier, and G. Roger, Phys. Rev. Lett. 49, 91 (1982).

[3] A. Einstein, B. Podolsky, and N. Rosen, Phys. Rev. 47, 777 (1935).

[4] Albert Einstein: Philosopher-Scientist, edited by P. A. Schilpp (Open Court, Peru, Illinois, 1970).

[5] A. Imamoglu, H. Schmidt, G. Woods, and M. Deutsch, Phys. Rev. Lett. 79, 1467 (1997).

[6] O. Firstenberg, T. Peyronel, Q.-Y. Liang, A. V. Gorshkov, M. D. Lukin, and V. Vuletić, Nature (London) 502, 71 (2013).

[7] J. Volz, M. Scheucher, C. Junge, and A. Rauschenbeutel, Nat. Photonics 8, 965 (2014).

[8] B. Gouraud, D. Maxein, A. Nicolas, O. Morin, and J. Laurat, Phys. Rev. Lett. 114, 180503 (2015).

[9] S. Ritter, C. Nölleke, C. Hahn, A. Reiserer, A. Neuzner, M. Uphoff, M. Mücke, E. Figueroa, J. Bochmann, and G. Rempe, Nature (London) 484, 195 (2012).

[10] W. B. Gao, A. Imamoglu, H. Bernien, and R. Hanson, Nat. Photonics 9, 363 (2015); H. M. Meyer et al., Phys. Rev. Lett. 114, 123001 (2015).

[11] J. Majer, J. M. Chow, J. M. Gambetta, J. Koch, B. R. Johnson, J. A. Schreier, L. Frunzio, D. I. Schuster, A. A. Houck, A. Wallraff, A. Blais, M. H. Devoret, S. M. Girvin, and R. J. Schoelkopf, Nature (London) 449, 443 (2007).

[12] M. A. Sillanpää, J. I. Park, and R. W. Simmonds, Nature (London) 449, 438 (2007).

[13] S. Barzanjeh, M. Abdi, G. J. Milburn, P. Tombesi, and D. Vitali, Phys. Rev. Lett. 109, 130503 (2012).
[14] J. Bochmann, A. Vainsencher, D. D. Awschalom, and A. N. Cleland, Nat. Phys. 9, 712 (2013).

[15] R. W. Andrews, R. W. Peterson, T. P. Purdy, K. Cicak, R. W. Simmonds, C. A. Regal, and K. W. Lehnert, Nat. Phys. 10, 321 (2014).

[16] J. Chan, T. P. M. Alegre, A. H. Safavi-Naeini, J. T. Hill, A. Krause, S. Gröblacher, M. Aspelmeyer, and O. Painter, Nature (London) 478, 89 (2011).

[17] J. D. Teufel, T. Donner, D. Li, J. W. Harlow, M. S. Allman, K. Cicak, A. J. Sirois, J. D. Whittaker, K. W. Lehnert, and R. W. Simmonds, Nature (London) 475, 359 (2011).

[18] A. D. O'Connell et al., Nature (London) 464, 697 (2010).

[19] T. P. Purdy, P.-L. Yu, R. W. Peterson, N. S. Kampel, and C. A. Regal, Phys. Rev. X 3, 031012 (2013).

[20] A. H. Safavi-Naeini et al., Nature (London) 500, 185 (2013).

[21] S. D. Bennett, N. Y. Yao, J. Otterbach, P. Zoller, P. Rabl, and M. D. Lukin, Phys. Rev. Lett. 110, 156402 (2013).

[22] J. Teissier, A. Barfuss, P. Appel, E. Neu, and P. Maletinsky, Phys. Rev. Lett. 113, 020503 (2014).

[23] A. Asadian, C. Brukner, and P. Rabl, Phys. Rev. Lett. 112, 190402 (2014).

[24] O. Romero-Isart, A. C. Pflanzer, F. Blaser, R. Kaltenbaek, N. Kiesel, M. Aspelmeyer, and J. I. Cirac, Phys. Rev. Lett. 107, 020405 (2011).

[25] D. E. Chang, J. I. Cirac, and H. J. Kimble, Phys. Rev. Lett. 110, 113606 (2013).

[26] A. Goban, C.-L. Hung, J. D. Hood, S.-P. Yu, J. A. Muniz, O. Painter, and H. J. Kimble, Phys. Rev. Lett. 115, 063601 (2015).

[27] M. Gullans, T. G. Tiecke, D. E. Chang, J. Feist, J. D. Thompson, J. I. Cirac, P. Zoller, and M. D. Lukin, Phys. Rev. Lett. 109, 235309 (2012). 